\title{
A Low-Cost Compliant Gripper Using Cooperative Mini-Delta Robots for Dexterous Manipulation
}

\author{
Pragna Mannam*‡, Avi Rudich ${ }^{* \ddagger}$, Kevin Zhang*‡, Manuela Veloso ${ }^{\dagger}$, Oliver Kroemer*, and F. Zeynep Temel* \\ ${ }^{*}$ Robotics Institute, ${ }^{\dagger}$ Machine Learning Department \\ Carnegie Mellon University, Pittsburgh, PA 15213 \\ *\{pmannam, arudich, klz1, okroemer, ztemel $\} @$ andrew.cmu.edu, ${ }^{\dagger}$ mmv@ @cs.cmu.edu \\ ${ }_{\ddagger}^{\ddagger}$ Equal Contribution \\ https://sites.google.com/view/mini-delta-robots
}

\begin{abstract}
Traditional parallel-jaw grippers are insufficient for delicate object manipulation due to their stiffness and lack of dexterity. Other dexterous robotic hands often have bulky fingers, rely on complex time-varying cable drives, or are prohibitively expensive. In this paper, we introduce a novel low-cost compliant gripper with two centimeter-scaled 3-DOF delta robots using offthe-shelf linear actuators and 3D-printed soft materials. To model the kinematics of delta robots with soft compliant links, which diverge from typical rigid links, we train neural networks using a perception system. Furthermore, we analyze the delta robot's force profile by varying the starting position in its workspace and measuring the resulting force from a push action. Finally, we demonstrate the compliance and dexterity of our gripper through six dexterous manipulation tasks involving small and delicate objects. Thus, we present the groundwork for creating modular multi-fingered hands that can execute precise and lowinertia manipulations.
\end{abstract}

\section{INTRODUCTION}

In unstructured settings like hospitals and homes, robots require the ability to execute dexterous manipulation tasks like handling delicate and small objects such as pills and coins. Many existing robotic end-effectors are designed for industrial applications where the focus is on repeatable and robust manipulation of large and rigid objects. However, these end-effectors can exert significant forces that can damage smaller, delicate, and nonrigid objects, like berries and playing cards. Interest in soft manipulators has grown recently because of their advantages in safety and compliance [1]. To leverage these desirable properties in dexterous manipulation, we propose a novel compliant gripper composed of cooperative 3-DOF mini-delta robots that are made using soft 3D-printed materials.

Delta robots are highly effective and accurate for pick and place tasks in a variety of industrial manufacturing and packaging processes [2]. However, utilizing them for other purposes has not been widely studied. In particular, many collaborative robot (cobot) arms used in both academia and industry are outfitted with either two finger parallel jaw grippers or vacuum grippers. Some labs and companies have used significantly expensive and complicated anthropomorphic (human-like) hands such as the shadow hand, which are typically difficult to control autonomously due to their high degrees of freedom [3]. Other researchers have developed their own hands such as soft pneumatic grippers [4] or jamming

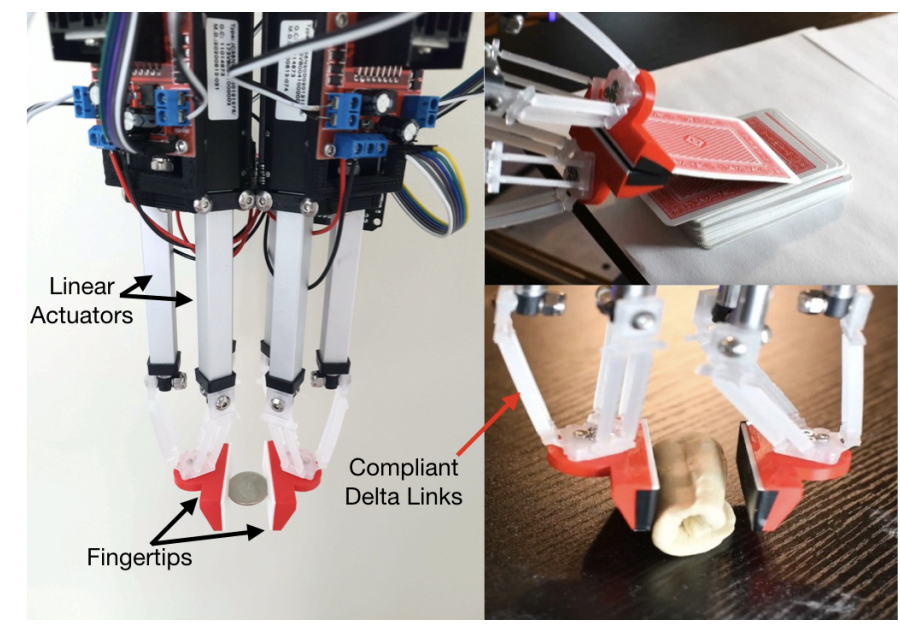

Fig. 1. Novel robotic gripper grasping a coin, a card, and a dough roll with two delta robots, each with three degrees of freedom and made from 3D-printed soft material, polypropylene (PP). The linear actuators, compliant delta links, and fingertips are shown for a pair of delta robots. The delta robot's links move up and down with the linear actuators fixed at the joints.

grippers [5], but they usually have a smaller workspace and less accuracy.

Our gripper, presented in this paper, consists of an endeffector with two 3-DOF delta robot modules as shown in Figure 1 In contrast to other grippers, our mini-delta robots use closed-form inverse kinematic solutions and soft materials which achieve high accuracy while still providing compliance. Furthermore, our end-effector is accessible through the use of 3D-printing and readily available off-the-shelf parts. The modular parts can be easily replaced and produced at a low cost. The price for our delta gripper is approximately $\$ 300$ ( $\$ 150$ per 3-DOF finger), which is significantly cheaper than the cost of some off-the-shelf grippers. For instance, the anthropomorphic Shadow Dexterous Hand starts from $\$ 50,000$ [6] and Robotiq's dexterous 3-finger adaptive robot gripper is $\$ 18,000$. Even a two-fingered gripper from Robotiq costs around $\$ 5,000$ [7]. The dexterous dynamixel claw from BAIR lab [8], which also has 3-DOF per finger, costs around $\$ 2500$ for 3-fingered manipulation. From our total delta gripper cost of $\$ 300$, the cost of the actuators ( $\$ 40$ each) is the largest cost out of all the delta robot materials. We therefore consider 
it a low-cost gripper when comparing the magnitude of cost with commercial dexterous grippers. Unlike these grippers, the delta robot gripper with its parallel mechanism is compliant, low-cost, easily manufactured, and modular.

Our main contribution in this paper is the design and modeling of a novel gripper composed of 3-DOF compliant delta robots. We start by presenting a design of the gripper and learn its kinematic model using neural networks as the traditional rigid delta model is inaccurate for compliant links. Subsequently, we construct a force profile of the compliant delta robot in various starting configurations using a one-axis force sensor. Finally, we conclude by using the gripper with the learned kinematic model on several dexterous manipulation tasks including manipulating a grape, aligning a pile of coins, picking up one coin from a pile, picking up a card from a deck, plucking a grape off of a stem, and rolling dough. Through these manipulation demonstrations, we present a multi-fingered hand design that can execute precise and lowinertia manipulations.

\section{RELATED WORK}

Delta robots are three translational degrees of freedom (DOF) parallel mechanisms that can be used for manipulation. The advantage of such a robot compared to a serial manipulator is that the inverse kinematics can be computed in closed-form, allowing for fast and easy control. Additionally, the motors are stationed at the base of the delta robot, creating a light end-effector that can move precisely with low inertia. Such low inertia mechanisms combined with compliant materials have a lower chance of harming objects and humans upon interaction. These qualities can be enhanced with the use of soft materials for a safe robotic gripper.

However, soft robots require a departure from classical methods for design, fabrication, and control [9]. The design of our compliant delta robot is similar to the laminate millimeterscaled delta robot [10] [11] and compliant parallelogram links characterized in [12]. Unique from these two approaches, we use 3D-printing for fabrication and linear actuators to create a prismatic delta rather than rotary motors for a revolute delta. Thus, we use three linear actuators per 3-DOF delta robot to move the end-effectors as shown in Figure 11 which behaves practically the same as the revolute delta [13].

Actuation and control of soft robots is an ongoing challenge. Some relevant works have tackled this by using learning-based methods. Truby et. al. [14] use deep learning to map piezoresistive sensor readings to $3 \mathrm{D}$ configuration of a complex soft robot. Homeberg et. al. [15] also use proprioceptive sensors but to distinguish objects in-hand. It is common to deduce where the robot is in space through external or internal sensors and in some cases both [16]. In our work, we exploit rigid delta kinematics to build a prior of where the delta end-effector is in space. Then, using a neural network, we create a robust model relating actuator positions to end-effector positions for delta robots of two different soft materials.

In our previous work on 3D-printed compliant delta manipulators [12], we characterized thermoplastic polyurethane

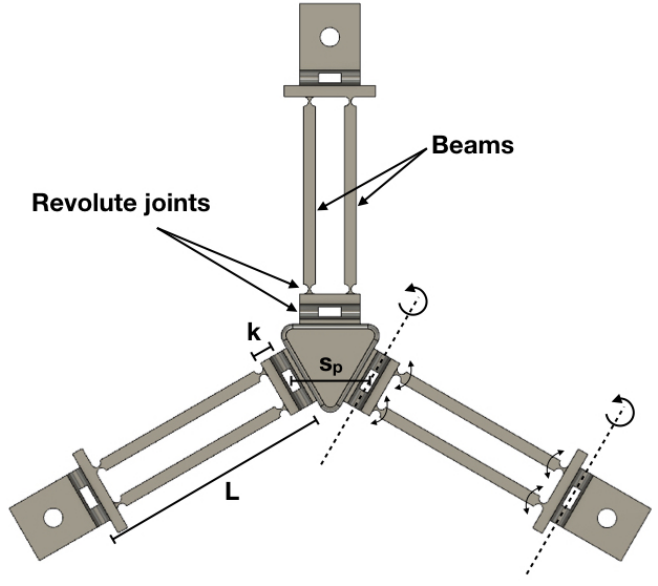

Fig. 2. This diagram illustrates the two orthogonal axes of rotation that approximate a universal joint and the measurements of $k, s_{p}$, and $L . k$ is shown as the distance between the two orthogonal axes of rotation, $L$ is shown as the distance between the axes of rotation at the top and bottom of a leg, and $s_{p}$ is shown as the distance between the the axes of rotation where the center of the parallelogram links attach to the end-effector. Each measurement represents a distance constraint between revolute joints in our simulation.

(TPU) and polypropylene (PP) parallelogram links for optimal design parameters. The main characteristic of a delta robot mechanism is that the end-effector stays parallel to the base. In our work, we use the optimal dimensions found to maintain this parallel relationship in [12] to design and fabricate a delta robot manipulator using the two materials, TPU and PP.

\section{Delta Manipulator Design}

Using 3D-printing and soft materials significantly impacted our design of the delta robot gripper. Our compliant delta links made from PP and TPU require a different design when compared to rigid delta links. The delta design, as shown in Figure 2, is also dependent on the gripper workspace and actuators. Additionally, fingertips made from PLA are mounted on the delta robot's end-effector, as shown in Figure 1. For comparison, out of the materials used, TPU is the most compliant, followed by PP and then PLA with tensile moduli of $26 \mathrm{MPa}$ (using ASTM D638), $220 \mathrm{MPa}$ (using ISO 527), and 2,346.5 MPa (using ISO 527), respectively [17]. We used an Ultimaker S5 to create all of our 3D-printed parts, but similar fused deposition modeling (FDM) printers are widely accessible, which greatly lowers the barrier of entry to make these low-cost compliant delta robotic manipulators.

\section{A. Setup}

In this work, we test delta robots made from two soft materials, PP and TPU, so we design two compliant delta links accordingly. We use compliant parallelogram links with living hinges to 3D-print delta robots as characterized in previous work [12]. The two living hinges rotate along orthogonal axes to approximate a universal joint in the delta links, as shown in Figure 2 Each leg is composed of two beams that move as a four bar parallel linkage mechanism, which transfer the 
motion from the linear actuators to the end-effector, as shown in Figure 1. These parallelograms have $0.375 \mathrm{~mm}$ hinges, and $2.5 \mathrm{~mm}$ and $4.5 \mathrm{~mm}$ thick beams for PP and TPU deltas, respectively. We use these values to ensure that the delta robot end-effector remains as parallel to the base, throughout as much of the robot's configuration space, as possible [12].

The delta links are attached to the ends of three ECO LLC Mini Electric Linear Actuators with $76.2 \mathrm{~mm}$ stroke and 20 $\mathrm{N}$ maximum load. The actuators are controlled through ROS serial with an Arduino Mega and L298N motor controllers. The total weight of the gripper with two delta modules is $1.03 \mathrm{~kg}$. The gripper is mounted on a Franka Panda Robot Arm for additional mobility during task executions. Further dimensions such as the length of the delta parallelogram beams (as shown in Figure 2) and distance between the beams are chosen according to the desired workspace of the delta gripper.

\section{B. Delta Actuators and Workspace}

The parallelogram links characterized in our previous work [12] were evaluated on revolute delta robots while our delta gripper uses prismatic deltas actuated by linear actuators. While the delta robot mechanism remains the same, the prismatic delta design allows more freedom in the packing of delta robots. Our gripper only features two delta robots as shown in Figure 1, although the design framework can be easily extended to more delta robots adjacent to the existing ones. Revolute delta robots would require more space around the rotary actuators, hence using linear actuators instead allows us to pack deltas closer together and enable cooperative capabilities between the robots.

The prismatic delta workspace is generally close to a hemisphere shape as shown in Figure 3. The workspace shown only accounts for joint angles until the delta links are perpendicular to the linear actuators, as significant deformation of the compliant links will occur after this point. We design adjacent delta robots such that they share a section of their workspace to enable cooperative manipulation of an object. Parameters such as the leg length $L$, distance between actuators at the base $s_{b}$, and distance between where the parallelogram links attach to the end-effector $s_{p}$ are chosen to create overlapping workspaces between adjacent deltas while taking into account the size of the linear actuators and how close we can pack them. Additionally, we use similar delta structure dimensions characterized in related work [12] except that the parallelogram beams were adjusted to be $6 \mathrm{~mm}$ apart from each other and $37 \mathrm{~mm}$ in length. These changes allow for larger joint angles and overlapping workspaces.

\section{Fingertip design}

For our delta gripper, we consider two types of fingertips 3 D-printed with $10 \%$ infill. First, the planar fingertips made from red Tough PLA shown in Figure 1 mimic a parallel jaw gripper in that the contact surfaces are flat and opposable. The second fingertip is spherical in shape and made from blue TPU as shown in Figure 7(d), making the compliance at the contact points significantly higher at $10 \%$ infill. Additionally,

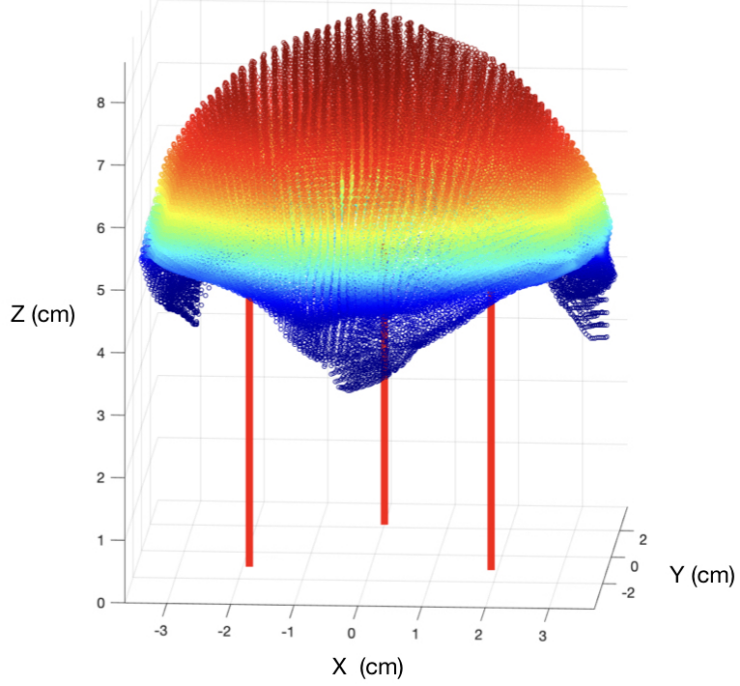

Fig. 3. The prismatic delta workspace, in centimeters, has a dome-like shape, where the robot can reach any of the points in $\mathrm{X}, \mathrm{Y}$, and $\mathrm{Z}$ axes. The three vertical red lines represent the position of the linear actuators. Colors are mapped to the height for a better visualization. The actuator lengths are limited to $4 \mathrm{~cm}$ so that no actuator can be above the delta's end-effector. Higher $\mathrm{z}$ values can be reached by adding a constant offset to every actuator.
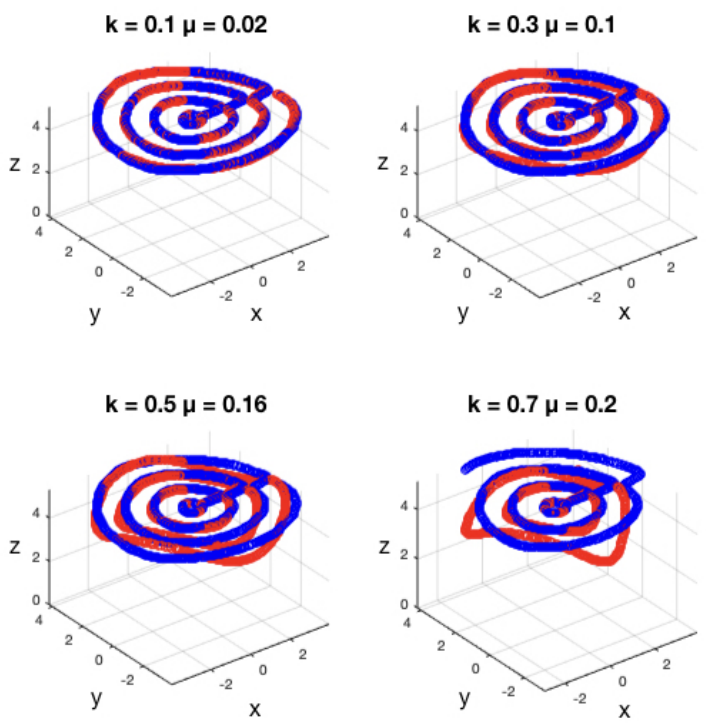

Fig. 4. Effects of varying the offset $k$ on the mean error $\mu$ from the path of a standard delta. The path of the delta with offsets is shown in red, and the path of a standard delta is shown in blue. For $k=.7 \mathrm{~cm}$, some actuator inputs become infeasible and the path is cut short.

the planar fingertips are padded with $2 \mathrm{~mm}$ thick foam and then electrical tape to increase friction and compliance between the fingertip and object. The main compliance of the gripper comes from the soft delta links as the foam padded fingertips are fully compressed after $1 \mathrm{~mm}$ of deformation. We exploit the compliance of our delta robots in conjunction with our fingertips to manipulate small and delicate objects dexterously. 


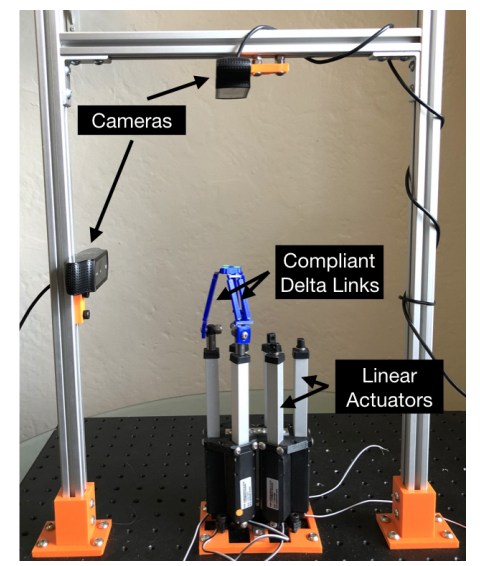

(a)

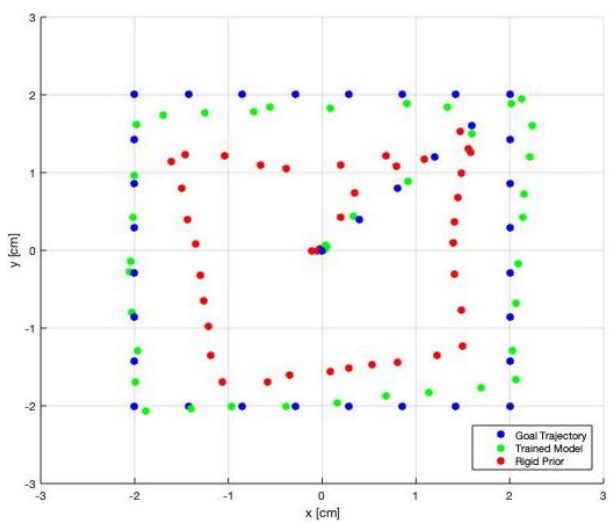

(b)

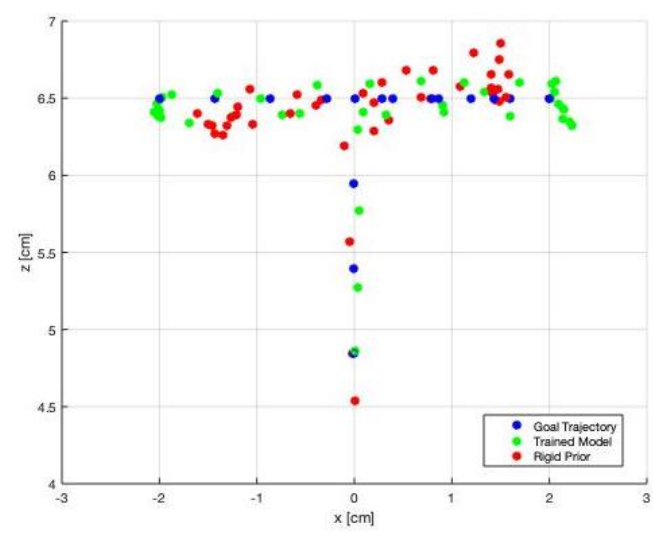

(c)

Fig. 5. (a) Marker-based stereo perception system to track the delta end-effector position using two orthogonal Logitech C920 cameras. The graphs compare measured and desired delta positions on the (b) XY and (c) XZ plane when following a test path (shown in blue) with the PP delta. The neural network trajectory prior to training is shown in red, and the trajectory after training is shown in green.

\section{Delta Modeling}

There is extensive literature analyzing rigid delta robots [18, 19], but the manufacturing of flexible delta robots through $3 \mathrm{D}$ printing introduces significant changes to the kinematics. We approximate each universal joint in the delta with an orthogonal pair of revolute joints that are separated by a small offset $k$ as labelled in Figure 2. We analyzed the effect of the offset between revolute joints on the delta workspace by modeling a rigid version of our delta in Simulink. To model the compliant delta kinematics, we use the rigid model as a prior and learn the residual correction using a distal learning approach with data obtained from a marker-based stereo visual tracker.

\section{A. Offset between Revolute Joints}

In order to approximate universal joints, our delta has orthogonal revolute joints that are separated by a small offset. We studied the effect of these offsets on the workspace by simulating a rigid version of our flexible delta robot. We measured four values from our delta robot to parameterize the simulation: leg length $L=4.8 \mathrm{~cm}$, distance between linear actuators $s_{b}=4.3 \mathrm{~cm}$, distance between where the legs attach to the end-effector $s_{p}=1.6 \mathrm{~cm}$, and an offset $k=.5 \mathrm{~cm}$ (see Figure 2).

In Figure 4 , we studied the effect of varying $k$ by measuring the deviation from the kinematics of a standard delta robot on a test trajectory. The test trajectory is a spiral that moves from the center of the workspace to its edge in the XY plane. The $\mathrm{z}$ coordinate is selected to maximize the width of the workspace. This path is then representative of the workspace as a whole because all points can be reached at other values of $\mathrm{z}$ by adding a constant offset to each of the three linear actuators. Figure 4 shows that for a large $k$, some actuator inputs become infeasible when the delta is near the edge of the workspace. Our value of $k=0.5$ warps the standard delta workspace by an average of $.16 \mathrm{~cm}$ on our test path, and does not cause any actuator inputs to become infeasible.

The most significant source of error when compared to the standard rigid delta is the deformation and twisting of the links. Each revolute joint in the delta applies a torque towards its rest position when actuated. In a rigid delta, this force is counteracted by the actuators, but for flexible deltas, equilibrium can be reached by changing the shape of the robot itself. Therefore, deformation occurs as joint angles become large. There are multiple ways that the robot is able to deform, and the type of deformation that occurs is determined by the robot's design parameters. Since we chose a high ratio of beam link width to joint hinge width in the parallelograms, the links themselves do not bend significantly. Instead, the revolute joints are able to twist a small amount, which can lead to large changes in the position of the end-effector.

\section{B. Learning Delta Robot Kinematics}

We used a marker-based stereo perception system to track the position of the real world delta and collect data to learn the forward and inverse kinematic models for the flexible delta robots as shown in Figure 5. To acquire the accurate kinematics, we trained a neural network that was pretrained to match the kinematics for our rigid delta model. We trained two different neural networks to model forward and inverse kinematics. Each network has 3 densely connected ReLU layers with 256 hidden units each and linear activation at the output.

The networks were trained using a distal teacher approach [20] where the forward kinematic model was trained to match input actuator positions with measured end-effector positions, and the inverse kinematics model learned inputs to the forward network that would reduce the error between its prediction and the measured position of the robot. This structure ensures consistency between the network outputs, and it makes it easy to identify and target areas where the kinematics are not wellknown because the two networks will produce conflicting 
results. The training data was also augmented using the symmetry of the delta robot. Observed end-effector positions were copied and reflected over the y-axis, corresponding to switching the heights of the two rightmost actuators in Figure 5. The actuator and end-effector positions for each observed point were also rotated by \pm 120 degrees about the z-axis. Finally, each observed end-effector position was given multiple $\mathrm{z}$ offsets, corresponding to adding a constant to the height of every actuator. These changes ensured that the learned workspace would be symmetrical.

Separate models were trained for both the TPU and PP deltas. The pretrained rigid delta model network had a mean error of $1.3 \mathrm{~cm}$ and $0.72 \mathrm{~cm}$ from the test path for TPU and PP deltas, respectively. To improve our pretrained network, it was sufficient to teach the network the flexible delta kinematics by running 100 trajectories that took approximately 20 seconds each. The models were then evaluated based on accuracy and repeatability when following a test path 50 times, as shown for PP deltas in Figure 5(b)-(c). After training on the TPU delta data, the mean error from the goal path was $0.33 \mathrm{~cm}$, and the mean pairwise error over 50 trajectories was 0.13 $\mathrm{cm}$. The mean error from the goal path for the PP delta was $0.28 \mathrm{~cm}$, and the mean pairwise error over 50 trajectories was $0.09 \mathrm{~cm}$. By fitting models to each type of delta, we were able to decrease the kinematics model error significantly for both deltas and confidently deployed them during our robot experiments.

\section{Force Profile}

As discussed in Section IV, deformation of the delta links occurs as joint angles become large, which happens towards the edges of the workspace. To determine whether this effect weakens the payload capacity of the delta in certain configurations, we displace the end-effector by a fixed distance along $\mathrm{X}$ and $\mathrm{Y}$ axes, and measure the resulting force to create a force profile of the delta.

For a given end-effector position $\left(X, Y, Z_{1}\right)$, another endeffector position $\left(X, Y, Z_{2}\right)$ may be achieved by offsetting all three of the linear actuators, as shown in Figure 6(a), by $Z_{2}-$ $Z_{1}$. We selected the $Z$ value that maximized the width of the delta workspace and calculated the force exerted by the delta gripper on the resulting XY plane. Any force measurement at a point $\left(X, Y, Z_{1}\right)$ is representative of the delta's force output at any other point $\left(X, Y, Z_{2}\right)$. We sampled the $\mathrm{x}$-axis in $4 \mathrm{~mm}$ increments and the $y$-axis in $5 \mathrm{~mm}$ increments from the point $x=-1.5 \mathrm{~cm}, y=-3.6 \mathrm{~cm}$ to the point $x=1.5 \mathrm{~cm}, y=$ $3.6 \mathrm{~cm}$. Accounting for symmetry, only positive $\mathrm{x}$-axis values are taken into consideration for measurements.

To test the force at a certain position of the workspace, the delta robot end-effector with a planar fingertip was moved to contact the load stem of a GSO-500 Transducer Techniques Load Cell. Then, we recorded the blocked force exerted by pushing the delta at the center of the fingertip surface $1,2,3,4$, and $5 \mathrm{~mm}$ into the load cell, as shown in Figure 6(a). Testing both TPU and PP delta robots, we observed that the force exerted grows linearly with the increased displacement. To measure the linearity, we calculated the $R^{2}$ value after linear regression for the five force measurements at each coordinate. On the TPU delta, the mean $R^{2}$ value across all measured points was 0.9541 , with a standard deviation of 0.1183 . The mean $R^{2}$ value for the PP delta was 0.9730 with a standard deviation of 0.0393 . This linear relationship allows us to control the force exerted by the delta robot through its displacement.

We grouped the data based on the $\mathrm{x}$ and $\mathrm{y}$ coordinates, and reported the mean blocked force when displacing the delta $5 \mathrm{~mm}$ in the direction of the load cell. Figure 6(b)-(c) shows that increasing the value of $x$ (moving parallel to the plane of the fingertip away from the center of the workspace) decreases the force output of the delta end-effector. There is no clear trend between the y coordinate of the delta and the mean force that can be exerted. This may be due to the orientation of the delta end-effector changing as it moves forward or backwards along the y-axis.

\section{EXPERIMENTS}

As a result of our work in Section IV, we are able to execute delta manipulator trajectories with precision. To further test the manipulator, we evaluate the success of manipulating various small objects with open loop control or human teleoperation using a PS4 Dualshock Controller. The six tasks we executed are as follows, 1) in-hand manipulation of a single grape, 2) aligning a pile of coins, 3) picking up a coin and rotating it in-hand, 4) slide-to-grasping a card from a deck, 5) twisting a grape off of its stem, and 6) rolling up dough between the fingers on a table. Unlike rigid manipulators, our soft delta gripper can exploit contacts, similar to a human hand, to execute tasks precisely. We chose these tasks to demonstrate the compliance of the deltas and their ability to manipulate delicate objects. While existing grippers may be able to execute these tasks using additional DOFs, we present a unique gripper that can perform all six tasks as a proof of concept. Due to our force profile experiments in Section V, we used the PP delta in all of our demos due to the higher force it can exert. All of the demos are shown in the accompanying supplementary vided ${ }^{1}$

In Figure 7(a), we show the delta gripper using planar fingertips to grasp a grape. Even when the delta robots use their maximum force to squish the grape, the compliance in the deltas prevent it from being crushed. Instead, the delta fingertips twist, while still holding on to the grape.

Next, in Figure 7(b), the deltas arrange a pile of coins by executing two parallel grasps that are orthogonal to each other. While this demo could also be completed by a parallel jaw gripper, there is a chance that the coins would fly out of the gripper if too much force was exerted on the pile. Our deltas gently align the pile of coins in order to create the precisely aligned pile. After the gripper aligns the coins in the pile, it grasps the top coin as in Figure 7 (c) and is able to rotate the coin in hand. This task illustrates the ability of our robot to

\footnotetext{
${ }^{1}$ https://youtu.be/yciJn3rgFHw
} 


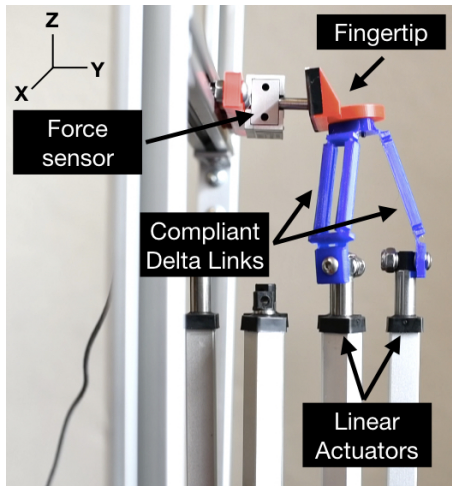

(a)

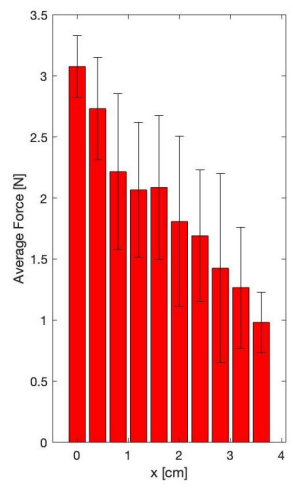

(b)

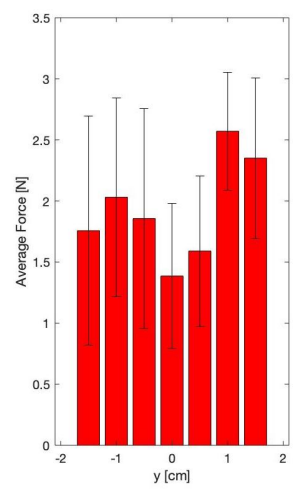

b)
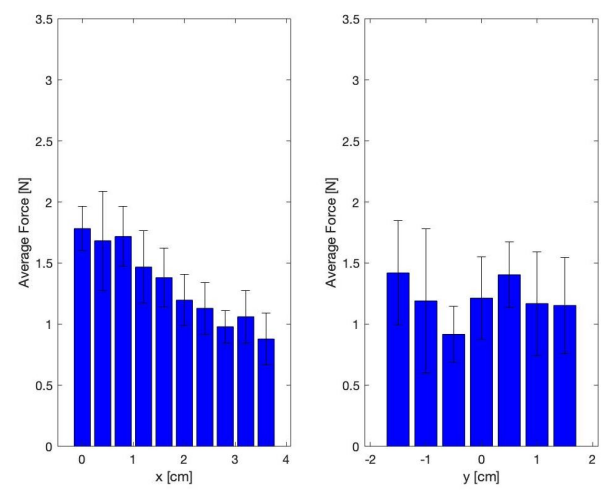

(c)

Fig. 6. (a) Force profile experiment setup consisting of a GSO-500 Transducer Techniques Load Cell and TPU delta robot with planar fingertip. The delta robot pushes on the load cell with a displacement of $5 \mathrm{~mm}$ at various positions in the workspace, along $\mathrm{X}$ and $\mathrm{Y}$ axes. The mean force exerted by the delta at different values of $\mathrm{x}$ and $\mathrm{y}$, and standard deviation are shown for the (b) PP deltas (c) TPU deltas.

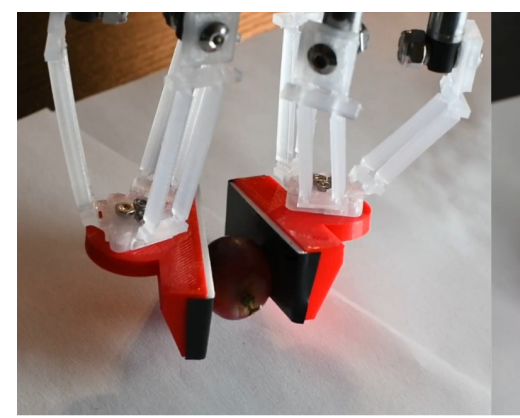

(a)

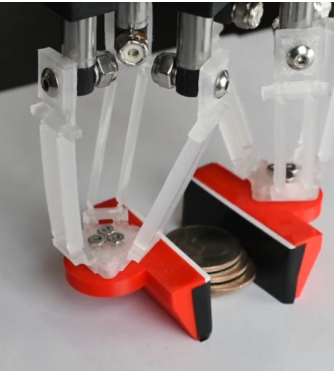

(b)

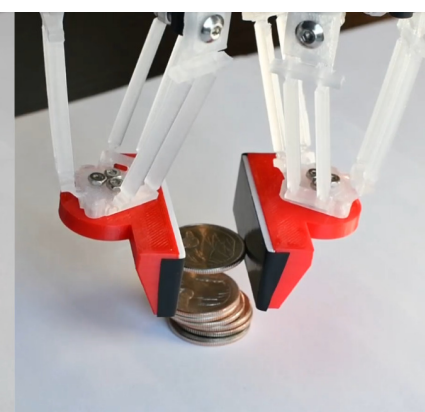

(c)

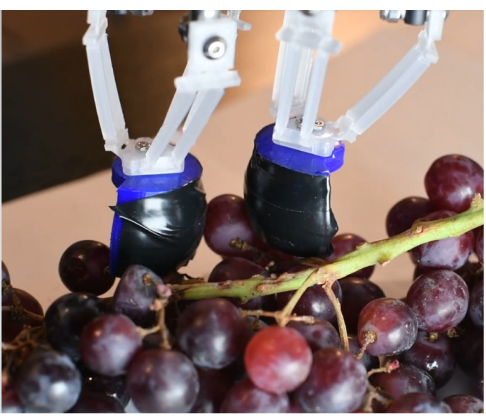

(d)

Fig. 7. Panels (a), (b), and (c) show the delta grippers with planar fingertips 3D-printed using PLA grasping a grape, aligning a pile of coins, and taking a coin from that pile and rotating it in hand, respectively. Panel (d) shows the deltas with spherical TPU fingertips picking a grape from its stem.

move in an additional axis that normal parallel jaw grippers cannot.

Using spherical TPU fingertips the delta gripper picks a grape off of a stem in Figure 7(d). Taking a grape off a stem requires the robot to twist the grape in order to apply the necessary pressure on the stem to get it to release without damaging the grape. The spherical fingertips allowed the grape to roll in between the fingertips, resulting in a twisting motion. Afterwards, the robot was able to remove the grape from the stem. This motion required human teleoperation as it involved positioning the fingertips so as to not allow other neighboring grapes to impede the motion of the deltas.

The final two tasks are illustrated in Figure 8 In the card pickup task, the top delta robot uses a stroking motion in order to slide the top card from the rest of the deck. Afterwards, the bottom delta lifts up and pinches the card together with the top delta to pick up a single card. The slide-to-grasp motion is made possible by the gripper's additional degrees of freedom and compliance. The sliding motion was programmed to execute autonomously, although it heavily depends on the initial positioning and orientation of the deltas relative to the cards.

The last task involved rolling a flat piece of dough into a spiral roll. This task also required human teleoperation due to the inherent compliance of the dough itself. One fingertip was used to mainly hold the dough in-place while the other was executing a scooping motion in order to get under the dough and push it. Without the degrees of freedom provided by the deltas, this task would likely be difficult for most grippers.

Throughout all of the demos, the compliance of the deltas and added degrees of freedom enabled a wider range of motion that normal parallel jaw grippers would not afford. In addition, as we had the inverse kinematics for the delta robots, we were able to quickly translate a desired trajectory into commands to the linear actuators. This direct mapping allowed us to easily teleoperate the robot with a PS4 Controller to complete tasks that would typically require a motion tracking hand setup in order to give the robot demonstrations [21]. In the future, we plan to explore more delicate and dexterous tasks with added sensors to provide feedback when interacting with objects.

\section{DISCUSSION AND CONCLUSION}

Through kinematic modeling, force profile characterization, and manipulation task executions, we explored the capabilities of compliant delta grippers made from two soft materials, TPU and PP. While the two materials vary significantly in compliance, the learned kinematic models for the TPU and PP deltas 


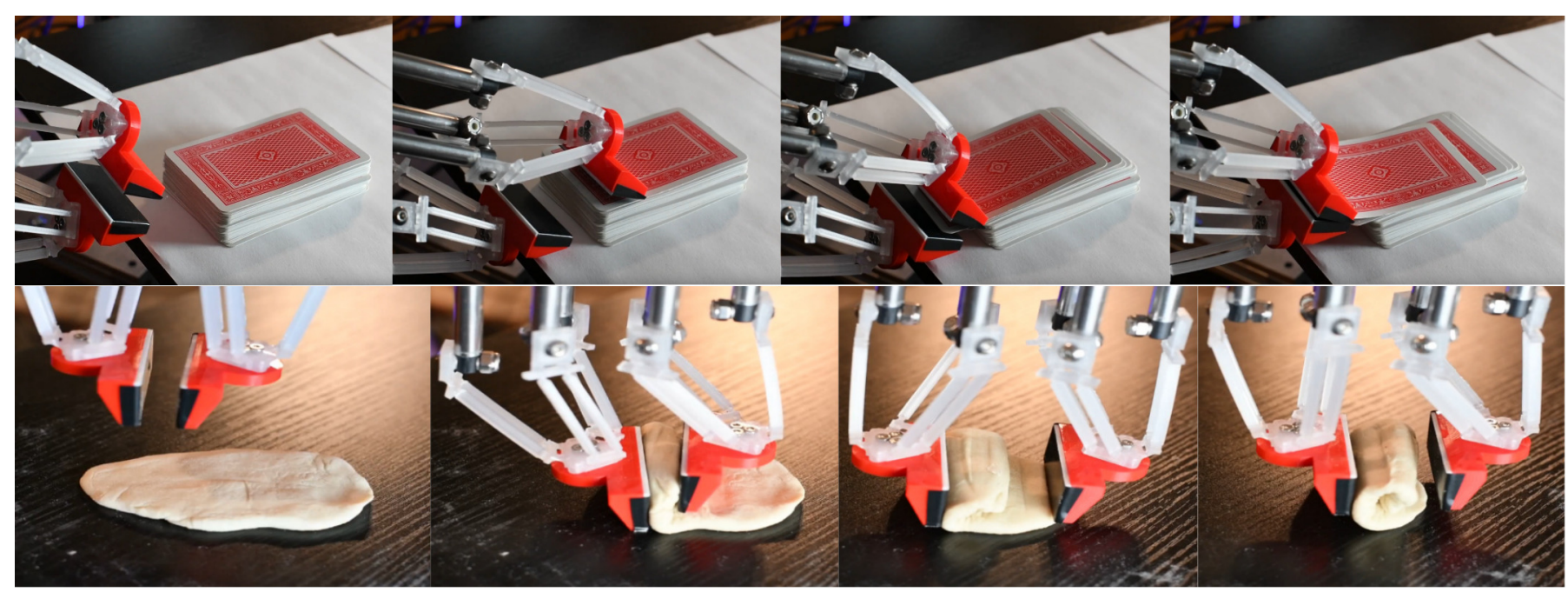

Fig. 8. Timelapse of the compliant delta gripper sliding the top card and picking it up from the deck, and rolling a flat piece of dough into a spiral.

did not differ significantly in performance. Additionally, the force profiles were similar in their ability to exert maximum forces at the center of the workspace. We expect similar trends to extend to delta robots made from materials similar to TPU and PP.

Our kinematic model learning error and force profile experiments show that the delta gripper is easy to control. The robot experiments show that dexterous manipulation tasks such as rolling dough and picking a grape off its stem can be executed with the degrees of freedom provided by each delta. Additionally, the compliance of the delta robot avoids damaging items like the grape. Thus, we can ensure that the delta robot can manipulate delicate objects and interact with its environment safely.

We present the groundwork for creating multi-fingered hands that can execute precise and low-inertia manipulations. Future extensions of this work can explore grasp planning and increasing the number of cooperative deltas to handle larger objects. In addition, we plan on incorporating internal and external sensors to the deltas in order to use visual and haptic feedback for more precise autonomous manipulation.

\section{ACKNOWLEDGMENTS}

The research presented in this work was funded by the National Science Foundation under Grant No. CMMI-2024794, Sony Group Corporation, and an Amazon Research Award. The research above does not reflect the opinions of our sponsors.

\section{REFERENCES}

[1] Cecilia Laschi, Barbara Mazzolai, and Matteo Cianchetti. Soft robotics: Technologies and systems pushing the boundaries of robot abilities. Science Robotics, 1(1), 2016.

[2] Jean-Pierre Merlet. Parallel robots, volume 128. Springer Science \& Business Media, 2005.
[3] OpenAI: Marcin Andrychowicz, Bowen Baker, Maciek Chociej, Rafal Jozefowicz, Bob McGrew, Jakub Pachocki, Arthur Petron, Matthias Plappert, Glenn Powell, Alex Ray, et al. Learning dexterous in-hand manipulation. The International Journal of Robotics Research, 39 (1):3-20, 2020.

[4] Josie Hughes, Utku Culha, Fabio Giardina, Fabian Guenther, Andre Rosendo, and Fumiya Iida. Soft manipulators and grippers: a review. Frontiers in Robotics and AI, 3: 69, 2016.

[5] John R Amend, Eric Brown, Nicholas Rodenberg, Heinrich $\mathrm{M}$ Jaeger, and Hod Lipson. A positive pressure universal gripper based on the jamming of granular material. IEEE transactions on robotics, 28(2):341-350, 2012.

[6] Shadow dexterous hand price list october $2020 . \quad$ https://www.shadowrobot.com/wpcontent/uploads/SHADOW-DEXTEROUS-HANDPRICELIST-OCT2020.pdf. Accessed: 2021-02-28.

[7] Robotiq. https://thinkbotsolutions.com/collections/ robotiq. Accessed: 2021-04-21.

[8] Henry Zhu, Abhishek Gupta, Aravind Rajeswaran, Sergey Levine, and Vikash Kumar. Dexterous manipulation with deep reinforcement learning: Efficient, general, and low-cost. In 2019 International Conference on Robotics and Automation (ICRA), pages 3651-3657. IEEE, 2019.

[9] Daniela Rus and Michael T Tolley. Design, fabrication and control of soft robots. Nature, 521(7553):467-475, 2015.

[10] Hayley McClintock, Fatma Zeynep Temel, Neel Doshi, Je-sung Koh, and Robert J Wood. The millidelta: A highbandwidth, high-precision, millimeter-scale delta robot. Science Robotics, 3(14), 2018.

[11] Jorge E Correa, Joseph Toombs, Nicholas Toombs, and Placid M Ferreira. Laminated micro-machine: Design 
and fabrication of a flexure-based delta robot. Journal of Manufacturing Processes, 24:370-375, 2016.

[12] Pragna Mannam, Oliver Kroemer, and F. Zeynep Temel. Characterization of compliant parallelogram links for 3dprinted delta manipulators. Proceedings of International Symposium on Experimental Robotics (ISER '20), March 2021.

[13] Mohamed Bouri and Reymond Clavel. The linear delta: Developments and applications. In ISR 2010 (41st International Symposium on Robotics) and ROBOTIK 2010 (6th German Conference on Robotics), pages 18. VDE, 2010.

[14] Ryan L Truby, Cosimo Della Santina, and Daniela Rus. Distributed proprioception of $3 \mathrm{~d}$ configuration in soft, sensorized robots via deep learning. IEEE Robotics and Automation Letters, 5(2):3299-3306, 2020.

[15] Bianca S Homberg, Robert K Katzschmann, Mehmet R Dogar, and Daniela Rus. Haptic identification of objects using a modular soft robotic gripper. pages 1698-1705, 2015.

[16] Gabor Soter, Andrew Conn, Helmut Hauser, and Jonathan Rossiter. Bodily aware soft robots: integration of proprioceptive and exteroceptive sensors. pages 2448 2453, 2018.

[17] Ultimaker: Materials. https://ultimaker.com/materials Accessed: 2021-02-27.

[18] M López, E Castillo, G García, and A Bashir. Delta robot: Inverse, direct, and intermediate jacobians. Proceedings of the Institution of Mechanical Engineers, Part C: Journal of Mechanical Engineering Science, 220(1): 103-109, 2006. doi: 10.1243/095440606X78263. URL https://doi.org/10.1243/095440606X78263.

[19] B. Mehrafrooz, M. Mohammadi, and M. T. Masouleh. Kinematic sensitivity evaluation of revolute and prismatic 3-dof delta robots. In 2017 5th RSI International Conference on Robotics and Mechatronics (ICRoM), pages 225-231, 2017. doi: 10.1109/ICRoM.2017.8466159.

[20] Michael I. Jordan and David E. Rumelhart. Forward models: Supervised learning with a distal teacher. Cognitive Science, 16(3):307-354, 1992. ISSN 0364-0213. doi: https://doi.org/10.1016/0364-0213(92) 90036-T. URL https://www.sciencedirect.com/science/ article/pii/036402139290036T

[21] Ankur Handa, Karl Van Wyk, Wei Yang, Jacky Liang, Yu-Wei Chao, Qian Wan, Stan Birchfield, Nathan Ratliff, and Dieter Fox. Dexpilot: Vision-based teleoperation of dexterous robotic hand-arm system. In 2020 IEEE International Conference on Robotics and Automation (ICRA), pages 9164-9170. IEEE, 2020. 